\title{
INTERCONSULTA EM ENFERMAGEM PSIQUIÁTRICA: QUAL A COMPREENSÃO DO ENFERMEIRO SOBRE ESTA ATIVIDADE?
}

\author{
Zeyne Alves Pires Scherer ${ }^{1}$ \\ Edson Arthur Scherer ${ }^{2}$ \\ Renata Curi Labate ${ }^{3}$
}

Scherer ZAP, Scherer EA, Labate RC. Interconsulta em enfermagem psiquiátrica: qual a compreensão do enfermeiro sobre esta atividade? Rev Latino-am Enfermagem 2002 janeiro-fevereiro; 10(1):7-14.

Nesta pesquisa objetivou-se identificar o conhecimento dos enfermeiros de serviços de clínica médica, cirúrgica e queimados, acerca da interconsulta em enfermagem psiquiátrica, através de uma entrevista semi-estruturada. As situações de assistência consideradas dificeis foram às relacionadas a recursos instrumentais e provisão de apoio psicológico. A maioria dos enfermeiros procura ouvir as queixas dos doentes, mas não sabe o que mais poderia fazer. Quando pedem ajuda, lembram de colegas, chefes, psicólogos e psiquiatras. Conhecem a especialidade do enfermeiro psiquiátrico, acreditam que possa ajudar com suporte emocional ao doente e à equipe. Consideram como interconsulta a avaliação do cliente por outro especialista.

DESCRITORES: referência e consulta, enfermagem psiquiátrica

\section{PSYCHIATRIC NURSING CONSULTATION AND LIAISON: HOW DO NURSES UNDERSTAND THIS ACTIVITY?}

This research aimed at identifying the knowledge of nurses working in medical and surgical clinics and with burned patients as to psychiatric nursing consultation by means of a semi-structured interview. Care situations considered to be difficult were related to instrumental resources and provision of psychological support. Most of the nurses try to listen to patients' complaints, but they do not know what else they could do. Whenever they need help, they think of colleagues, bosses, psychologists and psychiatrists. They know the specialty of the psychiatric nurse and believe that this professional can help with emotional support to the patient and the nursing team. They consider consultation and liaison to be an evaluation of the patient by another specialist.

KEY WORDS: referral and consultation, psychiatric nursing

\section{INTERCONSULTA EN ENFERMERÍA PSIQUIÁTRICA: CUAL ES LA COMPRENSIÓN DEL ENFERMERO SOBRE ESTA ACTIVIDAD?}

En ésta investigación se buscó identificar el conocimiento de los enfermeros de los servicios de medicina interna, cirugía y quemados, acerca de la interconsulta en enfermería psiquiátrica, a través de una entrevista semi-estructurada. Las situaciones de atención consideradas difíciles fueron las relacionadas con los recursos instrumentales y la provisión de apoyo psicológico. La mayoría de los enfermeros busca oír las quejas de los enfermos, pero no saben que más se puede hacer. Cuando piden ayuda, recuerdan compañeros, jefes, psicólogos y psiquiatras. Conocen la especialidad del enfermero psiquiátrico, y creen que puede ayudar con soporte emocional al enfermo y a el equipo. Consideran como interconsulta la evaluación del cliente por parte de otro especialista.

DESCRIPTORES: remisión y consulta, enfermería psiquiátrica

\footnotetext{
${ }^{1}$ Enfermeira Psiquiátrica, Doutoranda do Programa de Enfermagem Psuiquiátrica, Especialista em Laboratório da Escola de Enfermagem de Ribeirão Preto da Universidade de São Paulo, Centro Colaborador da OMS para o desenvolvimento da pesquisa em enfermagem, e-mail: scherer@eerp.usp.br; ${ }^{2}$ Professor da Universidade de Ribeirão Preto, Mestre em Ciências Médicas, Médico Psiquiatra do Departamento de Neurologia, Psiquiatria e Psicologia Médica do Hospital das Clínicas da Faculdade de Medicina de Ribeirão Preto da Universidade de São Paulo; ${ }^{3}$ Professor Doutor da Escola de Enfermagem de Ribeirão Preto da Universidade de São Paulo, Centro Colaborador da OMS para o desenvolvimento da pesquisa em enfermagem
} 


\section{INTRODUÇÃO}

$\boldsymbol{O}$ atendimento de enfermagem a indivíduos com doenças físicas caracteriza-se, atualmente, pela execução de procedimentos altamente técnicos disponíveis para a preservação da vida. Os profissionais envolvidos na assistência tendem a dar preferência à saúde física, deixando, muitas vezes, de abordar os aspectos emocionais.

A hospitalização é uma circunstância da vida que ocorre em qualquer idade e por diversas razões. Apesar das conotações negativas que possa ter, constitui-se num apoio social de referência ao qual se pode recorrer frente a uma situação de doença ou intercorrência que afete a integridade do indivíduo. Todavia, para a pessoa, representa um desafio à capacidade de adaptação, o que a faz reagir de diferentes maneiras. Trata-se de um processo que a equipe de saúde pode observar, no qual se destaca a influência dos fatores biopsicossociais que incidem na resposta do indivíduo à doença e/ou à internação. Tais fatores são, portanto, aplicáveis a inúmeras situações que envolvem a assistência a pacientes internados, sendo citados: pessoais - idade, sexo, personalidade, capacidade de enfrentamento do stress, experiências prévias, 0 significado da doença para a pessoa (perda, castigo, ganho, e outros), crenças e valores, habilidades para 0 auto cuidado e capacidade intelectual; sócio-culturais - o significado social da doença, a resposta da família, o status e o papel da pessoa, a situação de trabalho e econômica dela ao adoecer, os recursos sociais de apoio de que dispõe, e outros; relacionados à organização hospitalar a organização do serviço, as condições de assistência que se oferece, a adequação da planta física do local, a atitude da equipe de saúde frente ao paciente, a confiança que a mesma inspira, o estado físico/ emocional de seus integrantes; relacionados com a doença - os tipos de doença (aguda ou crônica), o prognóstico, as seqüelas ou incapacidades em potencial, e as medidas terapêuticas exigidas pela enfermidade ${ }^{(1)}$.

A resposta de um indivíduo à doença varia enormemente em função dele estar padecendo de uma enfermidade de curta duração (aguda), ou de longa evolução, podendo ser agravante 0 fato de o tratamento da mesma não ser resolutivo, mas, paliativo, mutilante, ou incapacitante. Nesta última condição situam-se as doenças crônicas ou intercorrências acidentais, sejam elas provocadas ou não, as quais obrigam a pessoa a modificar sua vida drasticamente, às vezes em definitivo ou por longo intervalo de tempo. Isto culmina na introdução de mudanças no estilo de vida pessoal, na dinâmica de relacionamentos familiar e social, assim como, em alterações na auto imagem e auto conceito do indivíduo provocado pela doença ou mesmo pelos efeitos dos próprios tratamentos como perda de peso, perda de cabelos, cansaço e outros ${ }^{(2)}$.
A este respeito em uma pesquisa com pacientes internados na unidade de queimados de um hospital universitário ${ }^{(3)}$ foi possivel observar que 0 tratamento restrito às úlceras, aos ferimentos, aos problemas físicos em geral, não parecia ser suficiente para a compreensão da doença e do doente. Havia um contexto maior no qual o enfermo é o veículo que traz a enfermidade e juntamente "carrega" os temores, a raiva, a ansiedade, a tristeza, a excitação, tornando o período de internação uma experiência traumática.

A compreensão da problemática "suporte emocional ao paciente com doenças físicas" pode ser facilitada com a consultoria psiquiátrica, a qual implica em uma abordagem que integra os processos fisiológicos, bioquímicos e seus distúrbios, considerando os três irredutíveis e inseparáveis fatores: biológico, psicológico e social. Tal postura retoma a concepção holística existente desde a antigüidade, apontada por Hipócrates ${ }^{(4-5)}$.

A psiquiatria de consultoria e ligação (consultation-liaison psychiatry) desenvolveu-se principalmente nos Estados Unidos, a partir da década de 30, com a abertura de unidades psiquiátricas em hospitais gerais. Cresceu no pós-guerra, com a internação de excombatentes com distúrbios psiquiátricos em tais hospitais ${ }^{(6)}$.

Os termos interligados, consultoria e psiquiatria, embora inter-relacionados, reconhecem dois aspectos distintos da cooperação entre a equipe psiquiátrica e as outras equipes especializadas. Consultoria refere-se, naturalmente, às situações em que os psiquiatras e/ou enfermeiros psiquiátricos fazem, a pedido de outro serviço, avaliações e recomendações quanto a pacientes ou problemas específicos. Psiquiatria de ligação refere-se a uma relação cooperativa contínua entre a equipe psiquiátrica e outras ${ }^{(7)}$.

Podemos encontrar na literatura o termo interconsulta psiquiátrica que tem sido empregado de forma genérica no nosso meio, em lugar de psiquiatria de consultoria e ligação, para indicar o conjunto de atividades desempenhadas por profissionais de saúde mental junto a diversos serviços do hospital geral ${ }^{(8)}$. Neste estudo optamos por utilizar o termo interconsulta ao invés de consultoria, para evitar a confusão com o termo consultoria do tipo empresarial ou administrativa prestada por profissionais da saúde a empresas, clínicas e outras instituições.

A interconsulta psiquiátrica é considerada uma área especial de conhecimento que se ocupa da assistência, ensino e pesquisa na interface da psiquiatria e demais especialidades da medicina. No Brasil, o número de serviços de interconsulta psiquiátrica é desconhecido e sua quase totalidade encontra-se em hospitaisescola. Na prática, os hospitais gerais, tendem a recusar a internação de pacientes sofredores de transtornos mentais ${ }^{(6)}$.

O movimento dos enfermeiros de saúde mental ocupando cargos em hospitais gerais na prática de interconsulta iniciou-se na década de 60 . A definição do papel interconsultor de enfermagem é 
dado pela Nursing Consultans Association (NCA) como sendo: "um enfermeiro que utiliza seus conhecimentos de enfermagem e sua experiência para promover cuidados de saúde através de meios distintos ao cuidado direto do paciente" ${ }^{\prime(9)}$.

Este atendimento indireto (interconsulta) $)^{(10)}$, por sua vez, não era suficiente para manejar a complexidade dos problemas apresentados por muitos pacientes originários das clínicas médica e cirúrgica. Solicitou-se, então, o envolvimento direto (ligação) dos enfermeiros interconsultores de saúde mental, pois, foi visto que sua participação mais próxima da equipe seria essencial na avaliação, planejamento de cuidados de enfermagem e na orientação dos profissionais a respeito do manejo das alterações psíquicas do doente.

A necessidade de enfermeiros interconsultores, por outro lado, vem aumentando pelo incremento da especialização entre profissionais da saúde, especificamente da enfermagem. Isto tem implicações diretas sobre a especificidade de cuidados nas distintas áreas de assistência, na crescente profissionalização da enfermagem, e com a necessidade dos enfermeiros resolverem os problemas referentes ao seu papel dentro de seu marco profissional. As vantagens da especialização em enfermagem psiquiátrica de interconsulta incluem o aperfeiçoamento da relação entre colegas de diferentes especialidades (consultor/consultante), intensifica a satisfação pessoal e profissional, e especializa e melhora a eficiência do cuidado ao paciente ${ }^{(9,11)}$.

0 interesse pelos aspectos emocionais dos indivíduos internados nas diversas clínicas de um hospital geral, aliado à nossa experiência enquanto profissionais atuantes na área da saúde, motivaram-nos a buscar elementos que nos permitissem estudar e conhecer a interconsulta de enfermagem psiquiátrica a partir da compreensão de enfermeiros assistenciais.

\section{OBJETIVOS}

Identificar a compreensão dos enfermeiros, atuantes em unidades de clínica médica, cirúrgica e de queimados, sobre a interconsulta em enfermagem psiquiátrica.

Buscar subsídios para sugerirmos, ou não, a participação do enfermeiro psiquiátrico no processo de interconsulta em hospital geral.

\section{MÉTODO}

Trata-se de um estudo do tipo exploratório descritivo, com abordagem qualitativa. Entendemos que esta abordagem parte do fundamento de que há uma relação dinâmica entre o sujeito e o objeto, um vínculo indissociável entre o mundo objetivo e a subjetividade do sujeito $^{(12)}$.

A população estudada foi composta por 6 enfermeiros, sendo dois da clínica médica, dois da cirurgia e dois dos queimados, da Unidade de Emergência do Hospital das Clínicas da Faculdade de Medicina de Ribeirão Preto da Universidade de São Paulo.

Em um primeiro momento foi necessária a obtenção do consentimento formal do Comitê de Ética e Pesquisa da Instituição. Além disso, contamos com o consentimento informal por escrito dos profissionais que participaram do estudo, obedecendo, assim, aos preceitos éticos e legais, assegurando-Ihes 0 anonimato $\mathrm{e}$ resguardando-lhes o direito, inclusive, de não concluir o estudo, se assim o desejassem.

Foi utilizado um roteiro de entrevista semi-estruturada (anexo) com questões norteadoras enfocando tópicos referentes as situações da assistência consideradas difíceis, manejo de aspectos emocionais dos clientes, enfermagem psiquiátrica e interconsulta. As entrevistas foram gravadas mediante consentimento dos sujeitos e posteriormente transcritas para análise dos dados. A coleta de dados foi realizada por um dos autores do estudo, nos horários em que os enfermeiros participantes se encontravam em suas respectivas unidades de trabalho.

Os dados foram submetidos à análise de conteúdo ${ }^{(13)}$ seguindo as etapas estabelecidas para tal. Este método constitui-se como uma técnica de pesquisa social utilizada para realizarmos uma descrição objetiva das informações dos sujeitos, tendo como meta à interpretação destes discursos.

\section{RESULTADOS E DISCUSSÃO}

Os profissionais, sujeitos do presente estudo, tinham de 3 a 25 anos de graduação em enfermagem. 0 tempo de trabalho dos mesmos nas unidades pesquisadas, variou entre 2 a 18 anos. Dois deles haviam feito especialização e um, pós-graduação.

A partir da análise temática das entrevistas, obtivemos as seguintes categorias:

\section{Dificuldades na assistência}

Dos 6 enfermeiros entrevistados, três (dois da seção de queimados e um da clínica cirúrgica) manifestaram dificuldades em lidar com aspectos relacionados aos fatores emocionais dos enfermos, evidenciados nos seguintes trechos das falas:

...aqui na seção de queimados que eu vejo é o lado psicológico. É o lado mais difícil de se lidar. Principalmente no queimado que se trata de dor... criança às vezes é dificil de se trabalhar com ela neste sentido... 
Sinto dificuldade na abordagem comportamental junto ao paciente e sua família.

Existem vários conceitos teóricos criados pela enfermagem salientando que a assistência ao ser humano deva ser globalizada ${ }^{(3)}$. Entretanto, a prática tem demonstrado que a aplicação desses pressupostos não é tão simples quanto possa parecer. Neste sentido, trabalhar com paciente em crise, como, por exemplo, o paciente queimado, requer do enfermeiro além do conhecimento técnico especializado, a habilidade em lidar com as emoções da pessoa doente.

Por sua vez, dois enfermeiros da seção de clínica médica e um da clínica cirúrgica apontaram, como dificuldades para a sua atuação, problemas relacionados com a infra-estrutura da instituição (falta de recursos materiais e humanos).

Às vezes, falta de material e pessoal e, às vezes, a falta de preparo do auxiliar, do enfermeiro.

Você... não pode fazer aquele plano de trabalho mais detalhado.. devido ser hospital de urgência... interrompe aquilo que você realmente desejava fazer.

Durante sua formação o enfermeiro tende a perceber a assistência ao paciente de forma fragmentada. Há uma dicotomia entre 0 discurso e 0 agir, entre a teoria e a prática, onde 0 aspecto expressivo da assistência de enfermagem é pouco desenvolvida em comparação ao aspecto instrumental, que continua tendo a maior atenção por parte do mesmo. Os enfermeiros valorizam a assistência e acreditam ser de sua responsabilidade 0 assistir emocionalmente. Apontam, contudo, dificuldades administrativas como entraves para que prestem a assistência emocional, além de outras de ordem pessoal, como: falta de experiência, despreparo acadêmico, medo de se envolver e insegurança frente à morte ${ }^{(14)}$.

Manejo dos aspectos emocionais do doente

Os profissionais sujeitos deste estudo tentam ouvir, mas não sabem o que podem fazer pelas pessoas que estão sob seus cuidados. Os enfermeiros da clínica cirúrgica e de queimados que falaram inicialmente sobre as questões emocionais da clientela como sendo dificuldades para sua atuação, sugerem a solicitação de auxílio especializado (psicologia, psiquiatria) para lidar com tais questões. Já os da clínica médica mostraram que à falta de recursos materiais e humanos, são considerados como fatores que complicam sua prática, buscam auxílio de suas chefias quando percebem que 0 doente necessita de suporte emocional.

Tento ouvi-las, a princípio, e tento de alguma forma analisar junto...na medida em que eu não consigo...peço ajuda de um colega para depois ir em busca de um profissional que realmente atue nesta área.

A maioria das vezes agente tem ouvido... é um desabafo da pessoa... acho que tem que encaminhar para a psiquiatria, para a psicologia. Eu tento conversar e entender o que está passando. Mas, é difícil. Então na maioria das vezes a gente pede acompanhamento da psiquiatria. Tento ajudar a entender o que esta acontecendo...Peço ajuda sempre ao diretor de enfermagem.

Como conseqüência da internação hospitalar, podem emergir problemas relacionados com a saúde mental dos pacientes que vão exigir da equipe de assistência uma abordagem abrangente dos mesmos. Deve ser dada, inclusive, uma atenção especial à aliança com o paciente e sua família, às conseqüências sociais da doença e, também, às dificuldades que outros profissionais da saúde possam vir a enfrentar no manejo de alguns indivíduos ${ }^{(15)}$

O tempo de permanência do paciente no hospital, em unidades de clínica médica ou cirúrgica, requer um envolvimento direto dos técnicos no suporte emocional. É sugerido, além disso, que para o profissional trabalhar adequadamente com 0 aspecto emocional do paciente precisa ter disponibilidade interna para tolerar as tensões provocadas pelas manifestações emocionais de dor e sofrimento dos outros, assim como, ter supervisão de um especialista para ajudá-lo a resolver situações que possam the parecer complicadas ou insolúveis ${ }^{(3)}$.

\section{Enfermagem psiquiátrica}

Todos os entrevistados responderam ter conhecimento da existência do especialista em enfermagem psiquiátrica. Um enfermeiro da clínica médica e um outro da cirúrgica atribuem a este profissional a função de atender doentes mentais. Acreditam, contudo, que o enfermeiro psiquiátrico poderia ajuda-los de alguma forma.

Já ouvi falar...dá suporte aos pacientes psiquiátricos, não só na parte física como também na mental...conversar com o enfermeiro é mais fácil do que com o médico, porque ele tem mais tempo.

Os demais entrevistados vêem o enfermeiro psiquiátrico como um técnico especializado que pode dar apoio psicológico para o doente, sua família e à equipe assistencial.

Já ouvi falar...acho que pode ajudar ...vai trabalhar o lado emocional do paciente e ajudando, também, toda equipe de enfermagem, fisioterapeuta, médico, a trabalhar todo este lado do paciente, o lado psicológico que a gente tem dificuldade em trabalhar.

Já ouvi falar... com certeza ajuda, inclusive a gente já trabalhou junto com o enfermeiro psiquiátrico aqui, ele ajuda muito nesta área, principalmente na unidade de queimados. É um profissional que eu considero extremamente importante...cuida da saúde mental dos nossos pacientes, suas famílias e dos membros da equipe. A enfermagem psiquiátrica atua na saúde mental dessas pessoas, no equilíbrio de emoções, de adaptações, de níveis de estresse, da compreensão desses conceitos que para nós ficam a desejar. 
$\mathrm{Na}$ literatura encontramos convergência entre os autores quanto ao entendimento de que o papel do enfermeiro é o de agente terapêutico e de que a base dessa terapia é o relacionamento estabelecido com o paciente a partir da compreensão do significado de seu comportamento. Na década de 60 o enfermeiro de saúde mental limitava-se às tarefas administrativas, de custódia e vigilância; porém, com o desenvolvimento da psiquiatria dinâmica, tornou-se um agente terapêutico, por sua possibilidade de influir nas relações interpessoais, de modificar favoravelmente 0 ambiente e de orientar as pessoas individualmente ou em grupos. É importante ressaltar que a comunicação e o relacionamento enfermeiro/cliente são considerados como base para a prática da enfermagem ${ }^{(16-18)}$.

Os entrevistados, apesar de não terem mencionado o termo interconsulta, sugeriram a presença do enfermeiro de saúde mental exercendo as funções atribuídas ao interconsultor. É sabido que 0 reconhecimento desta sub-especialidade tem crescido nos últimos anos. No que tange à capacitação do mesmo, o desempenho deste papel ${ }^{(19)}$ exige deste profissional, além do diploma de enfermagem psiquiátrica, conhecimentos clínicos e habilidade em lidar com a doença física e mental, a qual deve abranger desde as respostas normais até as manifestações da doença. A experiência combinada ajuda o enfermeiro interconsultor a transformar os princípios de saúde mental e suas recomendações, com aplicação imediata destes, a doentes de outras clínicas e à equipe de enfermagem.

Interconsulta

Uma das profissionais da unidade de queimados não soube definir o termo interconsulta, embora o tenha explicado nas questões referentes ao tema enfermagem psiquiátrica.

Interconsulta seria assim, é...logo que o paciente é admitido faria uma interconsulta? Não me lembro, estou meio confusa, eu tenho...eu não sei.

Já os outros entrevistados mostraram saber o que é a interconsulta. Um enfermeiro da clínica cirúrgica e outro da seção de queimados mencionaram tratar-se de um pedido de avaliação para um caso específico, respondido por um colega, não especificando a profissão do mesmo.

É o empenho do profissional na medida que ele é solicitado para avaliar e compartilhar de uma avaliação no seu seguimento de atuação num caso específico.

Você faz uma solicitação a um outro colega para que ele avalie um caso que você não tem tanto conhecimento quanto ele.

Um dos pesquisados da clínica cirúrgica reconhece a interconsulta médica junto ao paciente.

...é quando um profissional pede ajuda para outro...a ortopedia pede interconsulta para a psiquiatria para ajudar o paciente a sair da abstinência.
Os dois enfermeiros da clínica médica, apesar de referirem dificuldades com os aspectos instrumentais e de procurarem auxílio da chefia para lidar com as manifestações emocionais dos doentes, propõem que o enfermeiro psiquiátrico faça intervenção do tipo interconsulta direcionada aos pacientes e aos profissionais. Um sugere que o mesmo esteja junto à equipe (ligação), já o outro, que intervenha de forma indireta em outras clínicas (interconsulta).

...muitos pacientes aqui que tentam o suicídio, então, sempre eles pedem ajuda da psiquiatria...se tivesse o enfermeiro psiquiátrico para estar aqui mesmo...e para os funcionários também...se tivesse implantado isso no hospital ajudaria bastante em todos os sentidos.

...seria o enfermeiro psiquiátrico atuando dentro de uma área de clínica médica... intervindo aí como uma consulta aos pacientes e enfermeiros que estivessem no setor.

A sub-especialidade do enfermeiro interconsultor já é reconhecida como tal pelos americanos. Em 1990 a American Nurses Association definiu que a prática da interconsultoria de enfermagem psiquiátrica está baseada em teorias de estresse, enfrentamento e adaptação, com uma integração de perspectivas biológicas, psicológicas e sociológicas. Desta forma, portanto, enquanto o médico psiquiatra responde por drogas e tratamentos, os pedidos para a enfermagem são feitos mais comumente para suporte emocional para doente, família e equipe. $O$ interesse do médico é mais pela patologia, ao passo que o do enfermeiro é pela adaptação ${ }^{(20)}$.

Em uma revisão histórica ${ }^{(21)}$ foi reportado que os primeiros enfermeiros interconsultores trabalhavam exclusivamente com a equipe de enfermagem, auxiliando-a a entender as reações emocionais de seus pacientes através do processo educativo de solução de problemas. A interconsulta de enfermagem, de acordo com estes autores, tem entre suas características: a orientação para a equipe que cuida do paciente; o cuidado psicológico especializado direto para pacientes e suas famílias; o conhecimento sobre respostas normais e anormais à doença, e adaptação do paciente e família; 0 entendimento da inter-relação entre estados físicos e psicológicos; 0 conhecimento da teoria de sistemas e processo grupal; e a ligação entre as diferentes especialidades.

Os principais e mais usuais tipos de interconsulta na prática da enfermagem ${ }^{(9)}$ são: consulta centrada no cliente - enfoca a resolução de problemas imediatos do cliente, ajudando a confirmar o diagnóstico de enfermagem e a desenvolver planos de cuidados, tendo em conta não somente os conflitos detectados, mas, também, prevendo problemas potenciais; consulta centrada em problemas administrativos - utiliza suas habilidades de forma indireta, planificando programas, organizando seminários e grupos, e detectando áreas de trabalho estressantes, de maneira que redunde em uma maior competência profissional e em um melhor funcionamento da assistência; consulta centrada no enfermeiro e 
na equipe assistencial - o objetivo principal é melhorar 0 funcionamento da equipe de enfermagem ajudando-a, a compreender os fatores psicológicos da enfermidade do paciente e associá-los ao seu comportamento, clarificando e dando apoio para criar estratégias frente aos conflitos que podem surgir na equipe e ajudando os membros da equipe a utilizar os próprios conhecimentos e potencializar suas habilidades.

O enfermeiro interconsultor deve possuir conhecimentos específicos no campo em que é especialista e ser capaz de desenvolver atitudes que the permitam analisar as situações contextuais com objetividade. No caso do enfermeiro psiquiátrico interconsultor, um exemplo é o seu papel no processo deliberativo de enfermagem para decisões éticas sobre a remoção de tratamento de manutenção em pacientes terminais. Outro exemplo é o trabalho com o modelo cognitivo de tratamento, com o qual é possível resultado efetivo no manejo de pacientes, família e equipe utilizando tanto a abordagem individual como a grupal ${ }^{(22-25)}$

Em uma avaliação acerca da satisfação do enfermeiro de saúde mental interconsultor com relação aos pedidos que Ihes foram encaminhados em um hospital geral de Winnipeg (Canadá) ${ }^{(26)}$, foi notada a facilidade com que o consultante pôde ser notificado, a velocidade da resposta e a clareza com que suas formulações foram expressas. Houve satisfação dos interconsultores com a extensão de documentação escrita disponível e com a quantidade de seguimentos realizados.

Entre as dificuldades encontradas na interconsulta de enfermagem psiquiátrica ${ }^{(7)}$, destacam-se a existência de problemas na definição dos pedidos de interconsulta e que não são feitas solicitações em situações que assim o exigem. Existe, ainda, uma considerável relutância dos colegas em meios não psiquiátricos, bem como dos pacientes, em reconhecer a pertinência, ou até mesmo a existência de problemas emocionais e em pedir a assistência de um perito após se dar esse reconhecimento. Estes problemas seriam minimizados ${ }^{(7)}$ se existisse um serviço de interconsulta em enfermagem psiquiátrica estruturado no hospital geral.

\section{CONSIDERAÇÕES FINAIS}

Apesar de já estar definido o papel do enfermeiro psiquiátrico interconsultor nos Estados Unidos da América, identificando-o como uma sub-especialidade da enfermagem psiquiátrica e de saúde mental, e de na Inglaterra haver a atuação deste profissional na chamada interconsulta de ligação, no Brasil parece que esta técnica não tem até o momento sido proposta ou identificada.

Nesta pesquisa foi-nos possível comprovar que os colegas enfermeiros acreditam que podem encontrar auxílio e apoio com a participação de seus pares especialistas em psiquiatria junto ao seu trabalho. Eles sugerem que este colabore através de sua atuação direta (ligação) ou indireta (interconsulta) sobre os doentes e sobre a própria equipe. Portanto, os enfermeiros entrevistados referem-se ao enfermeiro de saúde mental interconsultor como uma possibilidade, parecendo não compreender exatamente sua função ou mesmo desconhecer a existência desta sub-especialidade.

No nosso entender o enfermeiro psiquiátrico interconsultor pode ter um papel semelhante ao dos psiquiatras, ou seja, pode responder a pedidos de consultas feitos por seus colegas de outras áreas, com atendimento direto ao doente, sua família ou mesmo oferecendo suporte para a equipe. Diferente do psiquiatra que se preocupa mais com a doença, 0 enfermeiro aborda a pessoa doente e sua adaptação. 0 interconsultor pode desempenhar, inclusive, a função de supervisor de equipes multidisciplinares (ou interdisciplinares) de saúde que procurem ou necessitem de tal abordagem

O potencial educativo é enfatizado ${ }^{(10,27)}$, salientando que ensinar é uma dimensão significativa da interconsulta de enfermagem em saúde mental, colaborando na orientação de membros novos da equipe de enfermagem, assim como nos programas de educação continuada, os quais devem focalizar-se principalmente no impacto emocional causado pela doença, tanto no paciente quanto na família.

Concordamos com os autores ingleses e americanos quanto à necessidade de treinamento ou especialização dos enfermeiros psiquiátricos para trabalhar em interconsulta como forma de capacitação dos profissionais e de reconhecimento da própria especialidade. 0 interconsultor necessita ter informações técnicas acerca da clínica na qual vai desempenhar seu papel, além de conhecimentos mais profundos em sua área específica de atuação (no caso enfermagem psiquiátrica). Quanto à formação técnica, os americanos sugerem a qualificação de mestre para habilitar um enfermeiro psiquiátrico interconsultor ${ }^{(20)}$. Acreditamos que para exercer esta função o enfermeiro psiquiátrico possa ter seu treinamento inserido nos cursos de especialização reconhecidos em nosso país. Além disto, propomos a inserção de disciplinas na graduação de enfermagem relacionadas ao tema interconsulta de enfermagem psiquiátrica.

$\mathrm{Na}$ enfermagem psiquiátrica brasileira, portanto, o serviço ou a prática de interconsulta é, ainda, obscuro, sendo reconhecida apenas a interconsultoria por parte dos médicos psiquiatras. Carecemos, desta forma, de esclarecimentos e de projetos de trabalho por parte dos colegas interessados no tema "interconsulta de enfermagem psiquiátrica", para que sua utilização seja viabilizada em termos práticos. Sugerimos que novas pesquisas sejam possibilitadas à partir dos achados deste estudo e das discussões e 
situações vivenciadas na prática diária dos enfermeiros assistenciais, não somente em serviços de urgência, mas em qualquer serviço de saúde. Ressaltamos a importância, portanto, da construção de um novo saber direcionado para este tema, adequado às nossas necessidades e realidades e de uma estrutura de ensino e avaliação constantes do mesmo.

\section{ANEXO}

\section{ROTEIRO DE ENTREVISTA}

1. Qual o tempo de formado (a)?

2. Qual a seção em que trabalha?

3. Há quanto tempo trabalha nesta seção?

4. Fez alguma especialização? Qual?

5. Que situações na assistência de enfermagem você considera mais difíceis?

6 . Como você lida com as pessoas que manifestam dificuldades emocionais

ou lhe trazem queixas de relacionamentos complicados ou outras histórias

\section{REFERÊNCIAS BIBLIOGRÁFICAS}

1. Lluch MT, Novel G, Royo MD. La enfermedad y la hospitalización. In: Martí GW, Canut MT, Vergara MDML. Enfermería: enfermería psico-social II. Barcelona: Salvat; 1991. p. 157-62.

2. Novel G, Lluch MTL, Vergara MDML. Enfermería: enfermería psicosocial II. Barcelona: Salvat; 1991.

3. Scherer ZAP. Percepções e significados atribuídos pelos pacientes à vivência da queimadura: a importância do processo interativo paciente-enfermeiro de saúde mental. [dissertação]. Ribeirão Preto (SP): Escola de Enfermagem de Ribeirão Preto/USP; 1995.

4. Meleiro AMAS. Abordagem do psicótico no hospital geral: interconsulta psiquiátrica. In: Miguel EC Filho, Fráguas R Junior, Meleiro AMAS, Assumpção FB Junior, editores. Psiquiatria e psicologia no hospital geral: a clínica das psicoses. São Paulo (SP): Mattavelli; 1992.

5. Navarro A. A interconsulta médico-psiquiátrica no hospital geral. Cadernos de Saúde da Comunidade 1992; 1(1):12-4.

6. Botega NJ, Dalgalarrondo P. Saúde mental no hospital geral: espaço para o psíquico. São Paulo(SP): Hucitec; 1993.

7. Kyes JJ, Hofling CK. Conceitos básicos em enfermagem psiquiátrica. Rio de Janeiro (RJ): Interamericana; 1985.

8. Shavitt RG, Busato G Filho, Miguel EC Filho. Interconsulta psiquiátrica: conceito e evolução. Rev Paul Medicina 1989; 107(2):108-12.

9. Cuadra AR, Rami XG. Salud mental. Processo de rehabilitación.

In: Cuadra AR, Apalategui MV, organizadoras. Enfermería: enfermería de salud mental y psiquiátrica. Barcelona: Salvat; 1991. p. 121-30.

10. Tringali RN. The role of the psyquiatric nurse consultant on a burn unit. Issues Mental Health Nurs 1982; 4(2):17-24.

11. Moschler LB, Fincannon J. Subspecialization within psychiatric consultation-liaison nursing. Arch Psychiatr Nurs 1992; 6(4):234-8. pessoais de suas vidas?

7. Você já sentiu vontade de pedir ajuda a algum colega ou outro profissional em alguma das situações que você citou? Em quem você pensou?

8. Você já ouviu falar no enfermeiro psiquiátrico?

9. Você acha que este profissional poderia ajudar de alguma forma?

10. O que você entende por enfermagem psiquiátrica?

11. O que você entende por interconsulta?

12. Chizzotti A. Pesquisa em ciências humanas e sociais. São Paulo (SP): Cortez; 2000.

13. Bardin L. Análise de conteúdo. Lisboa: Edições 70; 1991.

14. Ferreira NMLA. Assistência emocional: dificuldades do enfermeiro. Rev Baiana Enfermagem 1992; 5(1):30-41.

15. Goldman LS. O paciente aidético. In: Flaherty JA, Channon RA, Davis JM. Psiquiatria: diagnóstico e tratamento. Porto Alegre (RS): Artes Médicas; 1990. p.309-17.

16. Matheney R, Topalis M. Enfermería psiquiátrica. México: Interamericana; 1962.

17. Irving S. Enfermagem psiquiátrica básica. Rio de Janeiro (RJ): Interamericana; 1979.

18. Taylor CM. Fundamentos de enfermagem psiquiátrica. Porto Alegre (RS): Artes Médicas; 1992.

19. O'connor S. Enfermería psiquiátrica de enlace en un sistema de atención médica cambiante. In: Cassen NH. Psiquiatría de enlace en el hospital general. Madrid: Ediciónes Díaz de Santos; 1994. p. 74554.

20. Roberts D. Liaison mental health nursing: origins, definition and prospects. J Adv Nurs 1997; 25:101-8.

21. Nelson JKN, Schilke DA. The evolution of psychiatric liaison nursing. Perspect Psychiatr Care 1976; 14:60-65.

22. Dreyfus JK. Cognitive behavioral interventions in psychiatric liaison nursing practice. Med Psychother Int J 1990; 3:157-69.

23. Hart CA. The role of psychiatric consultation liaison nurses in ethical decisions to remove life-sustaining treatments. Arch Psychiatr Nurs 1990; 4(6):370-8.

24. Santmyer KS, Roca RP. Geropsychiatry in long-term care: a nurse centered approach. J Am Geriati Soc 1991; 39(2):156-159.

25. Samters J, Braun JV, Culpepper WJ, Cohen-Mansfield J. Description of a program for psychiatric consultations in the nursing home. Am J Geriatr Psychiatr 1994; 2(2):144-56. 
26. Newton L, Wilson KG. Consultec satisfacion with a psychiatric consultation liaison nursing service. Arch Psychiatr Nurs 1990; 4(4):264-70.
27. Ryan L. The consultative process in mental health nursing. In: Ryan L, organizador. Theories and concepts in the consultative process. US; 1979. p. 3-10. 